\title{
Need for payer-provider partnerships in addressing social determinants of health
}

\author{
Joseph T Kannarkat, MPP; James E Hartle, MD; and Natasha Parekh, MD, MS
}

\section{SUMMARY}

This article examines payer-provider partnerships in social determinants of health $(\mathrm{SDoH})$ interventions, identifies important factors for an approach centered around return on investment (ROI) using integrated delivery and finance systems as case studies, and advocates for increased collaboration between payers and providers when addressing SDoH.

Despite the numerous examples where payers and providers have attempted to independently address SDoH, there is limited evidence for the success of these interventions. Since most stakeholders individually do not have access to financial and clinical data, identifying an $\mathrm{ROI}$ for $\mathrm{SDoH}$ interventions is logistically challenging, but even when these data are available, stakeholders may not want to share their learnings due to negative findings and/or unwillingness to share proprietary information. These issues are further amplified by the effects of COVID-19 and its worsening effect on widening health disparities, but many payers and providers have risen to the challenge together.

This article advocates for the importance of payer-provider partnerships to address $\mathrm{SDoH}$ and uses examples of integrated delivery and finance systems as case studies of how these partnerships could function.

\section{Author affiliations}

Joseph T Kannarkat, MPP, University of Maryland School of Medicine, Baltimore; James Edward Hartle, MD, Geisinger Health System, Danville, PA; and Natasha Parekh, MD, MS, Queen's Health System, Honolulu, $\mathrm{HI}$.

\section{AUTHOR CORRESPONDENCE:} Joseph Kannarkat, 410.706.3100;

Joseph.kannarkat@som.umaryland.edu

J Manag Care Spec Pharm. 2021;27(6):791-96

Copyright $\odot 2021$, Academy of Managed Care Pharmacy. All rights reserved.
Payers and providers across the health care landscape are implementing approaches to address social determinants of health $(\mathrm{SDoH})$ with incentives to do so. SDoH are the conditions in which people are born, grow, live, work and age that shape health and include factors such as housing status, employment, transportation, access to food, and social support. ${ }^{1}$ Interventions addressing SDoH may improve patient health status and are a focus of significant financial investment for key stakeholders in health care. Although health care stakeholders have differing incentives in the health care stream, many have financial interests in addressing $\mathrm{SDoH}$. Payers, for example, are incentivized to reduce care utilization and costs of their insured populations, whereas providers aim to address the needs of their individual patients, which are often associated with SDoH. ${ }^{1-3}$ These interventions are becoming increasingly important in the COVID-19 era, which has exacerbated many unmet social needs. ${ }^{4,5}$ However, many interventions are often developed by stakeholders in siloes, likely limiting their potential for success.

We aim to examine examples in which payers and providers have sought to create SDoH interventions, identify important factors for an approach centered around return on investment (ROI) using integrated delivery and finance systems (IDFS) as case studies, and advocate for increased collaboration between payers and providers when addressing $\mathrm{SDoH}$.

\section{Payer and Provider SDoH Interventions}

In a January 2020 article from the New England Journal of Medicine, Finkelstein et al analyzed "hotspotting" at Camden Coalition of Healthcare Providers (CCHP), ${ }^{3}$ one of the most prominent hotspotting programs in the country. "Hotspotting" is a programmatic approach that aims to improve outcomes of patients who have high health care utilization by addressing their $\mathrm{SDoH}$ through health and social services. The CCHP hotspotting program began by enrolling patients while in the hospital and providing them with a multidisciplinary team consisting of nurses, social workers, community health 
workers, and health coaches. This team performed home visits, accompanied patients to primary and specialty care appointments, monitored blood pressure and sugar, provided disease-specific management education, and assisted with patient application to qualifying social service programs. Teams also addressed patient-specific challenges including organizing transportation to appointments when needed, finding accommodations for those with housing insecurity, and coordinating medication reconciliation to address adherence issues. Finkelstein et al concluded that the program did not seem to reduce 180-day readmissions more when compared with the usual postdischarge care, demonstrating just how difficult creating effective SDoH approaches and assessing their value can be. ${ }^{3}$

CCHP used hospital claims data to identify patients with high health care utilization, but this approach often provides an incomplete picture of unmet social needs. In a recent Health Affairs Blog article, Seidman et al speculate that additional data sources beyond hospital claims information, such as those from community partners, are likely needed to better identify and assess outcomes for patients requiring intensive intervention. ${ }^{6}$

As evidenced by the CCHP program, providers are generally the primary health care stakeholder to address $\mathrm{SDoH}$, since they traditionally lead the provision of health care and have direct relationships with patients. However, several stakeholders in the health care landscape are involved in addressing $\mathrm{SDoH}$, including the government, payers, and even drug manufacturers., ${ }^{1,7}$

Payers have incentive to ensure that $\mathrm{SDoH}$ for their beneficiaries are identified and addressed due to anticipated reductions in downstream utilization and costs. For example, UCare, a Minnesota-based insurer, partnered with the Amherst H. Wilder Foundation to address nutrition challenges in under-resourced communities with the Twin Cities Mobile Market Initiative in 2014. The Twin Cities Mobile Market is a grocery store bus that makes weekly stops selling subsidized healthy foods (eg, vegetables, fruits, dairy, and meats) to low-income areas in Minnesota's St. Paul and Minneapolis areas. In 2017, the program made 18,875 transactions that resulted in $\$ 215,000$ in net sales, an average of $\$ 11.39$ per transaction. Of 282 customers served, $87 \%$ indicate that the market provides access to healthy foods with a particular emphasis on fruits and vegetables. ${ }^{8}$

Although low-income populations are reached $37 \%$ of sales are from Electronic Benefits Transfer) through the market, the financial ROI and health-based effects are unclear. If $\$ 4$ is used to estimate the price of a single selfmade meal, less than 3 meals are consumed from the food purchased at the market per customer. Assuming that customers consistently buy food weekly (weekly bus schedule) and do not change their other dietary habits, it is unclear if the program ultimately improves the local population's nutrition. Fixed (ie, start-up costs) and variable costs (ie, cost of food) of the intervention were not reported, making it difficult to fully investigate their ROI. Furthermore, the program's leaders admit to difficulties offering high-quality foods at low prices and effectively managing the time of the program's staff. ${ }^{8}$

These challenges demonstrate the difficulty of developing sustainable payer-driven SDoH interventions. Despite a plethora of postings and press releases about other payer-driven $\mathrm{SDoH}$ interventions, including those through Medicare and Medicaid, ${ }^{1}$ literature on the ROI and clinical effect of these interventions is limited. This may be due to negative clinical results, limited cost effectiveness, limited ability to assess ROI, and unwillingness to share proprietary information.

\section{Integrated Delivery and Finance Systems as a Model}

Defining an ROI is challenging because of the ambiguous nature of evaluating the cost effect of an SDoH intervention. In a survey from Envolve to understand obstacles to SDoH interventions, $41 \%$ of 552 payer and provider respondents noted problems addressing $\mathrm{SDoH}$ for their respective populations, including budgetary constraints and unclear ROIs. ${ }^{9}$ Planned financial gains from SDoH programs with large sunk costs and revenue timelines that require multiple years to recoup losses often do not achieve financial ROIs in the short-term (eg, the same fiscal year). Social interventions can also vary widely in their cost-effectiveness and potential to isolate savings. These cost savings may include direct health benefits (eg, improvement of blood sugar levels and prevention of cardiac disease) in these calculations. Inclusion of these benefits is often inhibited by ability to financially capture costs and measure these benefits. Furthermore, there is a free-rider problem with these types of interventions, that is, a market failure when unintended beneficiaries from a communally accessible good or service do not need to contribute to have similar access. For instance, a payer can invest in food access programs, but realize only a fraction of those benefits if the beneficiary pool is larger than their constituency population or if the beneficiary pool disenrolls from their health insurance products. ${ }^{6}$ This discourages investment of health care stakeholders in these services, since there is an uncertain ROI.

IDFS, or systems that integrate the functions of both payers and providers, are uniquely positioned to address 
these challenges because of their structure and inherent ability to access necessary financial and clinical data to assess the comprehensive impact of value-based programs. For our purposes here, value-based approaches were considered a subset of typical cost-benefit approaches used to identify financial ROIs. Value-based programs differ from traditional cost-savings interventions by measuring financial savings associated with improved health metrics and including these metrics in the cost-benefit evaluation of these programs.

One such program example is the Cultivating Health for Success (CHFS) program from UPMC for You (an affiliate of the UPMC Health Plan) that began in $2010 .{ }^{10}$ Identifying that roughly 50 of its Medicaid or special needs plan members were homeless, UPMC sought to overcome challenges with lengthy wait lists for local housing programs and limited care coordination for its most marginalized members. UPMC partnered with Community Human Services, a local housing and urban development vendor, to integrate permanent housing, case management, and care coordination. The program offered services such as a secure living environment, social support, coordinated medical care, in-home assistance, consistent medical monitoring, and basic life skills training for 51 of 60 eligible participants over the first 5 years of the program. Roughly 24 members had to be successfully housed annually to offset the initial program cost of $\$ 150,000$. The program garnered an average overall cost savings of $\$ 6,384$ per housed member, demonstrating a potential value proposition for housing investment. Program data for 2015 and 2016 suggest that participating members had $42 \%$ fewer unplanned claims and a $1.5 \%$ increase in pharmacy costs, indicating medication adherence. ${ }^{10}$

Similarly, Geisinger is addressing food insecurity, rising diabetes prevalence, and treatment nonadherence through its Fresh Food Farmacy Program. Partnering with the Central Pennsylvania Food Bank, Geisinger seeks to improve healthy behaviors through food provision and provide comprehensive diabetes education in Shamokin, Pennsylvania. Participants are identified through surveys, medical record data, and Feeding America data. The program first provides healthy foods such as lean meats, whole grains, reduced fat dairy products, fruits, and vegetables to patients and their families. The program then aims to supplement these meals with nutrition education. Patients are assigned care teams consisting of a registered dietitian, registered nurse health manager, community health assistant, health coach, pharmacist, and pantry associate. Care teams attempt to cover patient gaps in care; monitor biometrics including weight, blood pressure, and hemoglobin A1c; and track medication adherence. The program is ongoing and expects
$\$ 8,000-\$ 12,000$ in care cost reduction for every 1-point improvement in A1c and an average cost of $\$ 3,500-\$ 4,000$ for patient food and nutrition education. Participants in the program's pilot averaged a 2-point improvement in A1c levels and were noted to experience fewer emergency department visits compared with those who were not enrolled. If this trend continues, Geisinger can expect a savings of $\$ 16,000-\$ 24,000$ savings annually for those who complete the program. Although the program is still in its early stages, the projected savings illustrate a large financial margin for the program if successful. ${ }^{11}$

\section{Applying IDFS Lessons to Payers and Providers}

What attributes make IDFS well suited to define a value proposition for $\mathrm{SDoH}$ ? The primary reason is their access to comprehensive, longitudinal financial and outcome data for patients, which has many advantages. A typical payer has access to treatment and cost data but has little insight into electronic health record-based clinical outcomes, whereas a provider has clinical outcome data but little insight into overall cost. An IDFS sees the patient's whole cost and regimen of care. In the Fresh Food Farmacy example, Geisinger's payer arm knows what services and treatments are being delivered but is only able to assess the patients' A1c levels by querying the provider arm. Furthermore, this structure allows an IDFS the ability to identify high users of care and pair that information with population-based care trends, which means that an IDFS is better suited to determine which $\mathrm{SDoH}$ traits are associated with higher utilization of care in its populations and address them. For example, UPMC for You, a Medicaid managed care organization, can identify costly patients as a payer but relies on UPMC's clinical partners, such as its providers and care coordination staff, to explore the housing status of its members. ${ }^{10}$

Interventions are rarely implemented perfectly; therefore, a capacity to pilot interventions and rapidly iterate on them is important. An IDFS is vertically coordinated for administrative, clinical, and financial management of patients, creating a test bed for piloting programs. Any value-based intervention requires selecting a target population, creating the actual intervention, defining the length of intervention, agreeing on metrics of evaluation, and considering how to modify the intervention in sight of new learnings. These are all critical steps in clarifying an ROI and require diverse information that most health care stakeholders do not individually have access to. Geisinger's Fresh Food Farmacy, for instance, identifies eligible patients through medical record data and questionnaires, tailors 
nutrition plans for patients via "prescriptions" by their primary care physicians, and longitudinally evaluates patient progress financially and clinically to determine an ROI. ${ }^{11}$ In a similar vein, since 2019, Kaiser Permanente has been working to connect its 12.3 million members to SDoH resources over the course of 3 years. Kaiser is working with Unite Us to track patients affected by negative $\mathrm{SDoH}$ and plans to expand real-time data analytics currently used in certain geographies to further reduce homelessness. ${ }^{12}$

Although the IDFS structure is not the only system type that allows for evaluation of an ROI for SDoH interventions, it illustrates the potential that arises from the synthesis of payer and provider information and from collaboratively working together to achieve common goals. Therefore, for nonvertically integrated systems, we urge payers and providers to partner in order to address $\mathrm{SDoH}$, since their strengths compensate for each other's weaknesses. For instance, a recent Marshall University study of federally qualified health centers $(\mathrm{FQHC})$ in rural Appalachia examined use of community health workers (CHW) for chronic care management. Originally provider driven, the study successfully engaged 2 payers to sustain the CHWs through memoranda of understanding with $2 \mathrm{FQHCs} .{ }^{13}$

In another notable example, Humana began its Social Determinants of Health Value-Based Program in partnership with Ochsner Health to address challenges such as food insecurity, loneliness, and housing instability in March 2020. ${ }^{14}$ Partnerships such as these can financially align incentives to address $\mathrm{SDoH}$ through payer-provider payment models.

\section{Addressing SDoH During COVID-19}

COVID-19 has amplified the importance of addressing SDoH, since SDoH are highly implicated in COVID-19 outcomes. According to the World Economic Forum, 500 million people could fall into poverty, with many more experiencing a drastic drop in income. ${ }^{4}$ Patients with social determinantbased issues such as homelessness, unemployment, and poor social support are more likely to become infected by COVID-19 and experience severe symptoms and higher mortality rates. ${ }^{4-6}$ It is also well documented that those with $\mathrm{SDoH}$ issues are secondarily more likely to experience issues with heightened mental health crises and uncontrolled chronic disease management. ${ }^{15,16}$

As such, the pandemic is acting as a catalyst for health policy and system change to improve access to high-quality health care and overcome SDoH-based issues. ${ }^{4}$ For example, the Centers for Medicare \& Medicaid Services have relaxed restrictions for reimbursement of telehealth visits, creating opportunities to provide safe and convenient access to care without patients having to worry about transportation and taking time off of work or childcare to seek health care services. ${ }^{17}$ Many of these relaxed restrictions are likely to continue beyond COVID-19. ${ }^{18}$

Private payers and health care systems have similarly sought opportunities to address $\mathrm{SDoH}$ during the pandemic. Blue Cross Blue Shield of Oklahoma, for instance, is repurposing its "Caring Van" fleet, which usually provides immunizations and screenings to thousands of children in underserved communities, to provide needed COVID-19 testing to underserved communities with limited access to care. ${ }^{2}$ Southern Humboldt Community Healthcare District (SoHum Health), a provider organization in California, is using hospital staff and volunteers to address the needs of 3,500 community seniors by having them assist with acquiring groceries and medications. ${ }^{19}$ Sohum Health has mobilized this group to use a 16-question script and action plan to identify the SDoH-based needs of their residents and leverage the support of the local health department, emergency services, Chamber of Commerce, banks, fire departments, and service groups to address these needs.

These types of interventions are encouraging first steps for payers and providers across the country, and policymakers should continue this momentum by encouraging increased collaboration between various health care stakeholders to ensure that social services are delivered to appropriate populations and that disparities are not further amplified through the pandemic. ${ }^{4}$

\section{Payer-Provider Partnerships to Address SDoH in the COVID-19 Era}

The current economic downturn and social restrictions resulting from COVID-19 has and will continue to prevent key services from reaching the marginalized. Luckily, payers and providers have partnered to rise to the occasion. For instance, CareOregon (an Oregon-based Medicaid managed care plan) and providers have partnered to distribute flip phones and smart phones to members to ensure that they have access to increasingly popular telehealth services. Additional services offered by CareOregon through its providers include transit passes for health-related reasons and air conditioners. ${ }^{20}$ CareFirst (a Blue Cross Blue Shield plan in Virginia) has also partnered with local health departments and hospitals in Maryland, Washington DC, and northern Virginia to identify vulnerable COVID-19 populations. To secure appropriate medication and resources, the insurer has already identified 180,000 at-risk members based on their clinical conditions, social situations, education, and use of community resources. ${ }^{2}$ 
In spite of these encouraging signs, COVID-19 will have an effect on the capacity of organizations to invest in SDoH. Although large hospitals are better equipped financially to weather the pandemic than that of physician-based, rural, and safety-net organizations ${ }^{21}$ all of these organizations are likely to become increasingly risk averse to negative ROIs. Therefore, partnerships with payers may offer an opportunity to spread the risk burden for interventions and make them more attractive options to pursue.

Also, payers and providers should be encouraged to share the outcomes of their SDoH programs. Having successful frameworks to learn from will limit unnecessary trial and error in intervention development and better elucidate challenges involved in program creation. Although COVID-19 presents issues to health care stakeholders across the board, it may also be an opportunity to address these barriers that limit the spread and adoption of creative approaches to unmet social needs.

There is a need for payer-provider collaborations to identify the right approach for investment in SDoH. ${ }^{9}$ Although there are still many needed efforts to identify the "special sauce" for constructing and demonstrating the value of SDoH interventions, there is exciting work being performed by stakeholders throughout the health sphere to address these challenges via partnerships. For now, it remains the next big hurdle to tackle in the evolution of population health.

\section{DISCLOSURES}

No outside funding supported the writing of this article. Hartle is employed by Geisinger Health System. The other authors have nothing to disclose.

\section{ACKNOWLEDGMENTS}

The authors thank Mr. W. Thomas McGough, Jr. (UPMC) for his help in the preparation of this article.

\section{REFERENCES}

1. Artiga S, Hinton E. Beyond health care: the role of social determinants in promoting health and health equity. Kaiser Family Foundation. May 10, 2018. Accessed June 9, 2020. https://www. kff.org/disparities-policy/issue-brief/ beyond-health-care-the-role-of-socialdeterminants-in-promoting-health-andhealth-equity/

2. America's Health Insurance Plans. Health insurance providers are innovating to address socioeconomic needs during COVID-19 and beyond. June 2020. Accessed August 7, 2020. https:// www.ahip.org/wp-content/uploads/ Socioeconomic-Needs-Innovation-Brief. pdf

3. Finkelstein A, Zhou A, Taubman S, Doyle J. Health care hotspotting - a randomized, controlled trial. N Engl J Med. 2020;382(2):152-62. doi:10.1056/ NEJMsa1906848

4. Shah GH, Shankar P, Schwind JS, Sittaramane V. The detrimental impact of the COVID-19 crisis on health equity and social determinants of health. J Public Health Manag Pract. 2020;26(4):317-19. doi:10.1097/PHH.0000000000001200

5. Abrams EM, Szefler SJ. COVID-19 and the impact of social determinants of health [comment]. Lancet Respir Med. 2020;8(7):P659-661. doi: 10.1016/ S2213-2600(20)30234-4

6. Seidman J, Bardin R, Napoles A, Khan M. Look beyond hotspotting to focus on a broader population's unmet social needs. Health Affairs Blog. July 10, 2020. Accessed August 8, 2020. http:// www.healthaffairs.org/do/10.1377/ hblog20200702.63975/full/

7. Take Action for Health. Pilot study report. May 2017. Accessed June 18, 2020. https://www.takeactionforhealth.org/ pilot-study-report
8. Wilder Foundation. Twin Cities Mobile Market. April 16, 2018. Accessed December 5, 2020. https://www.wilder. org/wilder-research/research-library/ twin-cities-mobile-market

9. Envolve. Strategies for payer-provider collaboration around social determinants of health. Accessed June 9, 2020. https://www.envolvehealth.com/oursolutions/social-determinants-health/ strategies-for-payer-collaboration-socialdeterminants-health.html

10. Modern Medicaid Alliance. UPMC for You-cultivating health for success. July 6, 2017. Accessed March 21, 2020. http://modernmedicaid.org/ medicaid solutions upmc for you/

11. Hess A, Passaretti M, Coolbaugh S. Fresh Food Farmacy. Am J Health Promot. 2019;33(5):830-32. doi:10.1177/0890117119845711d

12. Bresnick J. Kaiser Permanente launches full-network social determinants program. Health IT Analytics. May 6, 2019. Accessed June 18, 2020. https://healthitanalytics.com/news/ kaiser-permanente-launches-full-network-social-determinants-program

13. Crespo R. An emerging model for community health worker-based chronic care management for patients with high health care costs in rural Appalachia. Prev Chronic Dis. 2020;17:E13. doi:10.5888/ pcd17.190316

14. Humana Healthcare. Humana launches innovative value-based program to address social determinants of health. March 4, 2020. Accessed March 21, 2020. https://press.humana.com/news/ news-details/2020/program-to-addresssocial-determinants-of-health/default. $\underline{\operatorname{aspx} \# \mathrm{gsc} . \mathrm{tab}=0}$

15. Pfefferbaum B, North CS. Mental health and the COVID-19 pandemic. N Engl J Med. 2020;383(6):510-12. doi:10.1056/NEJMp2008017

16. Mirsky JB, Horn DM. Chronic disease management in the COVID-19 era. Am J Manag Care. 2020;26(8):329-30. doi: 10.37765/ajmc.2020.43838 
17. Shachar C, Engel J, Elwyn G. Implications for telehealth in a postpandemic future: regulatory and privacy issues. JAMA. 2020;323(23):2375-76. doi:10.1001/jama.2020.7943

18. Wicklund E. FTC weighs in on expanding CMS telehealth coverage beyond COVID-19. mHealthIntelligence. June 5, 2020. Accessed August 9, 2020. https://mhealthintelligence.com/news/ ftc-weighs-in-on-expanding-cms-telehealth-coverage-beyond-covid-19
19. American Hospital Association. Case study: getting in front of COVID-19: addressing social determinants of health to save the lives of seniors. Accessed August 9, 2020. https://www.aha.org/ case-studies/2020-04-07-case-studygetting-front-covid-19-addressing-socialdeterminants-health

20. CareOregon. Provider support: health-related services. 2020. Accessed August 8, 2020. http://careoregon.org/ providers/support
21. Bleser WK, Singletary E, Crook H, Gonzalez-Smith J, McClellan MB, Saunders R. Maintaining progress toward accountable care and payment reform during a pandemic, part 1: utilization and financial impact. Health Affairs Blog. April 14, 2020. Accessed August 8, 2020. https://www.healthaffairs.org/ do/10.1377/hblog20200410.281882/full/ 